\title{
TRANSESTERIFICAÇÃO DO ACETATO DE ETILA COM METANOL: AVALIAÇÃO TERMODINÂMICA
}

\author{
A. F. A. OLIVEIRA ${ }^{1,2}$, P. V. QUEIROZ ${ }^{1}$, C. H. F. CUNHA ${ }^{1}$, A. G. SATO ${ }^{1}$, F. A. \\ RODRIGUES $^{1}$ \\ ${ }^{1}$ Universidade Federal de Viçosa, Departamento de Química \\ ${ }^{2}$ E-mail: ana.f.alves@ufv.br
}

\begin{abstract}
RESUMO: A transesterificação é uma das mais primordiais reações da química orgânica, e é responsável pela síntese de diversos produtos, nos mais variados ramos industriais. $O$ estudo aqui realizado consiste em avaliar o comportamento da reação modelo de transesterificação de acetato de etila com metanol a fim de se obter acetato de metila $e$ etanol usando a abordagem de minimização de energia de Gibbs para cálculo de equilíbrio químico. A simulação foi realizada no software Aspen Plus 8.8 e para efeitos comparativos variou-se as temperaturas de reação e razões molares de reagentes. A partir dos resultados de simulação nota-se que a temperatura de reação não exerce grande influência na composição de equilíbrio. Por outro lado, o equilíbrio termodinâmico mostra forte dependência na razão molar de reagentes quando se analisa o processo isotermicamente.
\end{abstract}

PALAVRAS-CHAVE: Transesterificação; Simulação; Condições de Reação; Termodinâmica

\section{INTRODUÇÃO}

A obtenção de ésteres pode ocorrer através da reação entre um ácido carboxílico e um álcool (esterificação) e da reação entre um éster e um álcool na presença de catalisador formando um novo éster e um novo álcool (transesterificação) (Peng et al., 2013). Os ésteres orgânicos ocorrem de forma natural em óleos, gorduras, flores e frutas sendo responsáveis por odores e sabores característicos e em consequência disso uma grande variedade de ésteres é empregada com o objetivo de conferir sabor e odores artificialmente a diversos produtos; tornando-se assim um produto de interesse para as indústrias de perfumaria, de alimentos, de cosméticos e farmacêutica, além de serem espécies intermediárias nas indústrias de plásticos e de química fina (Alonso et al., 2009; Tomke, Rathod, 2015).

O equilíbrio químico e termodinâmico de uma reação corresponde a composição na qual produtos e reagentes minimizam a energia livre de Gibbs do sistema. Para se obter as composições de equilíbrio de uma dada reação, deve-se solucionar a formulação matemática que minimize a energia de Gibbs do sistema (Leal et al. 2016). 
As reações de transesterificação podem ser empregadas em vários setores da indústria, como, por exemplo, a produção de biodiesel. Para qualquer reação de transesterificação, os parâmetros que irão influenciar no equilíbrio da reação são semelhantes, tais como o tipo de álcool e a razão molar álcool:éster, temperatura, pureza de reagentes, agitação do meio reacional, a natureza do catalisador e a dosagem, se é ácido ou básico, se é homogêneo ou heterogêneo, entre outros. (Encimar et al., 2002).

As reações de transesterificação são reações reversíveis e, por isso, é importante deslocar a reação a favor da formação dos produtos, uma das maneiras é utilizar o excesso de álcool. Os álcoois mais utilizados nas reações de transesterificação são o metanol e etanol, os quais podem ser obtidos de fontes renováveis.

O estudo aqui realizado consiste em avaliar o comportamento da reação modelo de transesterificação de acetato de etila com metanol a fim de se obter acetato de metila e etanol, segundo a reação 1 :

$$
\begin{aligned}
& \mathrm{CH}_{3} \mathrm{COOC}_{2} \mathrm{H}_{5}+\mathrm{CH}_{3} \mathrm{OH} \rightleftharpoons \mathrm{CH}_{3} \mathrm{COOCH}_{3}+\mathrm{CH}_{3} \mathrm{CH}_{2} \mathrm{OH} \\
& \text { Acetato de Etila Metanol Acetato de Metila Etanol }
\end{aligned}
$$

A composição de equilíbrio de uma dada reação possui propósito singular nos processos industriais, dessa forma, surgem várias pesquisas de otimização dos métodos utilizados para solucionar as equações de equilíbrio químico e de fase, através da minimização de energia de Gibbs (Yancy-Caballero e Guirardello, 2013).

\subsection{Avaliação termodinâmica}

De acordo com Derevich et. al. (2006), pode-se calcular a composição do equilíbrio de um sistema através do método de minimização de energia de Gibbs, pois, segundo Néron et. al. (2012), um sistema químico isotérmico e isobárico está em equilíbrio quando a energia de Gibbs é mínima. Nesse método é necessário conhecer apenas a composição dos componentes alimentados ao sistema, a pressão, a temperatura e os produtos reacionais.

Em um sistema multicomponente, a energia de Gibbs pode ser expressa em função da temperatura $(T)$, energia de Gibbs padrão $\left(\Delta G_{\mathrm{f}_{n}}^{0} \mathrm{i}\right)$, quantidade de matéria, p.ex. em mols $\left(\mathrm{n}_{\mathrm{i}}\right)$ e atividade de cada componente (âa $)$, como está escrito na equação 2.

$$
\mathrm{nG}\left(\mathrm{n}_{\mathrm{s}} \mathrm{T}_{\mathrm{z}} \mathrm{P}\right)=\sum_{\mathrm{i}=1}^{\mathrm{M}} \Delta \mathrm{G}_{\mathrm{f}_{\mathrm{s}} \mathrm{i}}^{0} \mathrm{n}_{\overline{\mathrm{i}}}+\mathrm{RT} \sum_{\mathrm{i}=1}^{\mathrm{M}} \mathrm{n}_{\mathrm{i}} \ln \hat{\mathrm{a}}_{\mathrm{i}}
$$


Assim, para obter os valores das composições no equilíbrio, é necessário minimizar a equação 2. Para isso, deve-se derivar a equação 2 com relação aos componentes e igualar a zero (equação 3).

$$
\left(\frac{\partial \mathrm{nG}}{\partial \mathrm{n}_{\mathrm{i}}}\right)_{\mathrm{T}_{z} \mathrm{P}_{z} \mathrm{n}_{\mathrm{i}} \neq \mathrm{n}_{\mathrm{i}}}=0
$$

O sistema deve respeitar o balanço de massa, ou seja, o somatório do número de átomos de cada componente $\left(\mathrm{n}_{\mathrm{i}} \cdot \beta_{\mathrm{i}}\right)$ após o equilíbrio deve ser igual ao número de átomos adicionados ao reator $\left(b_{j}\right)$ (equação 4$)$.

$$
\sum_{\mathrm{i}=1}^{M} n_{i} \beta_{i}=b_{j}
$$

Utilizando as equações 3 e 4 é possível obter a composição do sistema no equilíbrio, conhecendo apenas os produtos e reagentes da reação e a composição inicial do sistema.

\section{MATERIAIS E MÉTODOS}

Para realizar a simulação da reação aqui estudada utilizou-se o software Aspen Plus ${ }^{\circledR}$ 8.8 cuja licença está disponível nos computadores do Laboratório de Simulação de Processos Químicos e Catálise (LaSiP) do Departamento de Química da Universidade Federal de Viçosa.

No Aspen Plus ${ }^{\circledR}$ avaliou-se o sistema reacional utilizando um reator de Gibbs, que utiliza o princípio de minimização da energia de Gibbs para fornecer as concentrações de cada componente do sistema ao atingir o equilíbrio químico da reação, e utilizou-se o pacote termodinâmico UNIFAC que reproduz a não-idealidade de líquidos e possível formação de fase gasosa (Yancy-Caballero, Guirardello, 2013). A representação para simulação computacional do sistema reacional usando reator de Gibbs é mostrada na Figura 1.

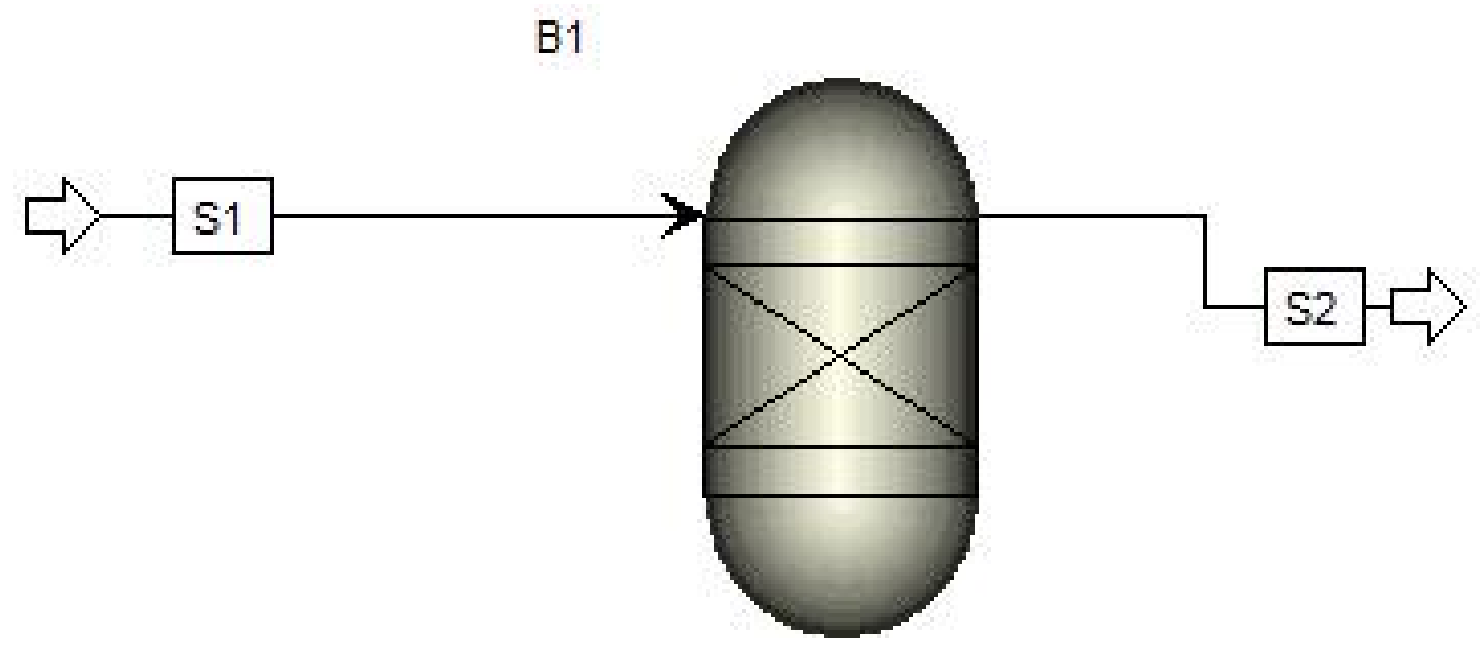

Figura 1 - Representação para simulação usando abordagem de minimização de Gibbs. 
O reator de Gibbs do software apresenta condições de fluxo contínuo, dessa forma, a corrente de entrada do reator de Gibbs utilizado é denominada S1 e é composta pelos reagentes da reação aqui estudada, e a corrente de saída do reator S2, é composta pelos produtos da reação e a parcela dos reagentes não convertidos. O catalisador não é utilizado para a simulação, uma vez que, este elemento não é consumido na reação, e os cálculos do programa são feitos apenas observando-se a termodinâmica envolvida.

Para avaliar o comportamento da reação no equilíbrio, variou-se alguns parâmetros do reator e da corrente de entrada. Inicialmente avaliou-se a influência da temperatura na conversão e composição no equilíbrio, variando-a na faixa de $173,15 \mathrm{~K}$ a $373,15 \mathrm{~K}$ e com base nos estudos realizados por Marciniuk (2012) e Castro (2013) manteve-se constante a razão molar de 1:6 de acetato de etila:metanol e a pressão constante igual a 1bar.

Em outra análise, manteve-se a temperatura constante em 293,15K e variou-se a razão de reagentes da corrente de entrada do reator e observou-se a variação da conversão no equilíbrio para uma pressão constante igual a 1 bar.

\section{RESULTADOS E DISCUSSÃO}

Os resultados encontrados para as quantidades molares das espécies no equilíbrio termodinâmico, através da simulação da reação de transesterificação do acetato de etila com metanol, em um reator de Gibbs, variando-se a temperatura de reação utilizando uma razão molar constante de 1:6 de acetato de etila:metanol e pressão constante de 1bar é mostrado na Figura 2.

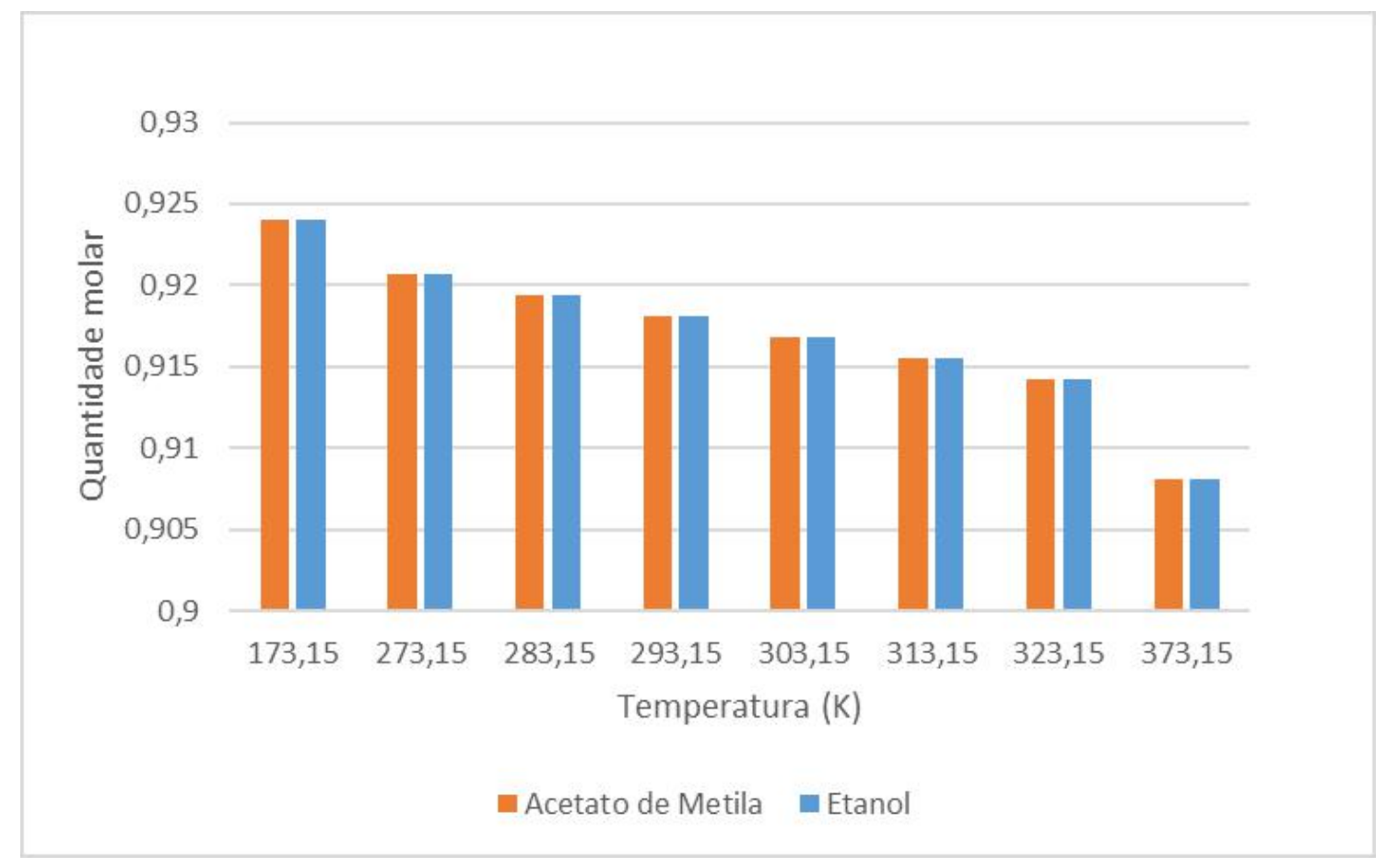

Figura 2 - Efeito da variação da temperatura sobre a quantidade molar das espécies no equilíbrio e a conversão no equilíbrio obtidos pelo software Aspen Plus 8.8. 
Analisando a Figura 2, fica evidente a pouca influência da temperatura na conversão final no equilíbrio. Em uma região onde varia-se a temperatura em uma faixa de $173,15 \mathrm{~K}$ a $373,15 \mathrm{~K}$, houve uma variação da conversão no equilíbrio de pouco menos de $2 \%$.

Contudo, a análise termodinâmica em questão não especifica o tempo reacional necessário para que o sistema atinja esses valores de equilíbrio calculados para cada temperatura. Então apesar de não indicar uma alteração substancial nos valores de equilíbrio, a temperatura, pode promover uma cinética reacional mais rápida permitindo que o sistema atinja o equilíbrio termodinâmico.

Quando se variou as razões molares dos reagentes utilizados, mantendo a temperatura constante igual a $293,15 \mathrm{~K}$, obteve-se as quantidades molares de cada uma das espécies no equilíbrio permitindo a comparação entre o reagente limitante da reação e o produto de interesse. O efeito da variação da razão molar é mostrado na Figura 3.

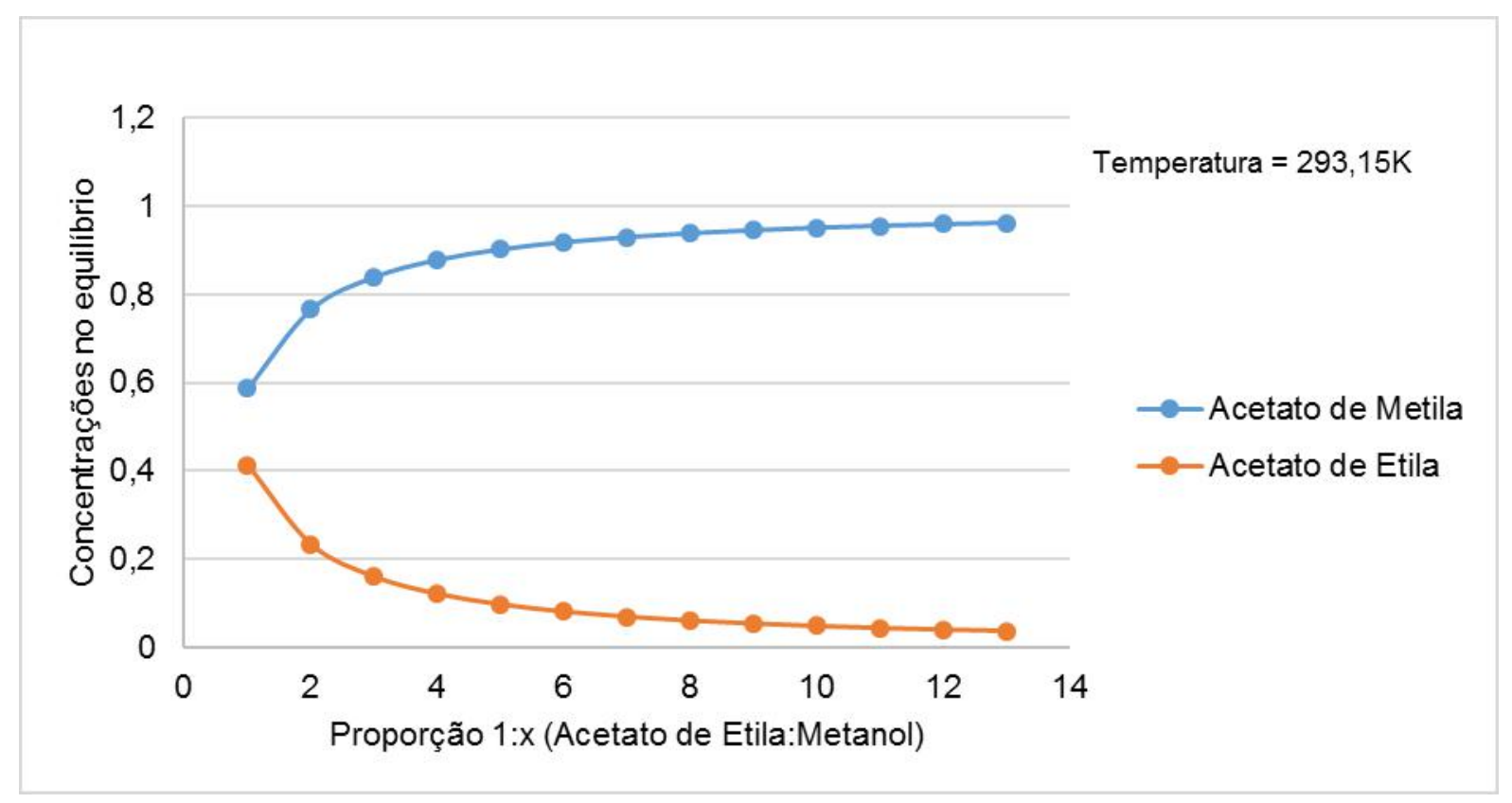

Figura 3 - Efeito da variação da razão molar de reagentes sobre a quantidade molar das espécies no equilíbrio reacional obtidos no software Aspen Plus 8.8.

De acordo com os resultados da Figura 3, que correspondem à simulação com alteração nos valores de razão molar, a conversão final no equilíbrio tende a aumentar com o aumento da razão molar dos reagentes.

Quando se compara os valores de razão molar e quantidade das espécies no equilíbrio, nota-se que é possível atingir uma conversão maior quando se aumenta a razão de metanol. Entretanto, para valores de razão molar superiores a 1:6, a composição do equilíbrio não 
aumenta de forma substancial e por isso não torna viável a sua utilização por despender maiores gastos com insumos.

Segundo a simulação termodinâmica, ao se utilizar a razão molar 1:6 a conversão no equilíbrio é de 91,82\%, no entanto as pesquisas de Marciniuk (2012) e Castro (2013), que utilizam esta razão obtiveram resultados inferiores para a conversão, mostrando que os experimentos práticos não necessariamente conseguem reproduzir a conversão simulada.

Outra condição que poderia ser estudada é o efeito da pressão sobre a conversão final das espécies em equilíbrio termodinâmico pois ao se manter constante a temperatura, a energia de Gibbs molar de excesso depende da composição da mistura e pressão. Porém a influência da pressão só é considerada para pressões altas, uma vez que, a pressões baixas ou moderadas o efeito é suficientemente pequeno para poder ser desprezado (Prausnitz et al., 1986 apud Yancy-Caballero, 2012). No entanto, ao se pensar em uma aplicação futura em processos industriais a utilização de altas pressões gera um alto gasto com utilidades que pode inviabilizar o processo e em consequência disso não se realizou a variação da pressão no reator.

\section{CONCLUSÕES}

A simulação termodinâmica apresenta os valores ideais de conversão no equilíbrio para as diferentes temperaturas e razões molares avaliadas da transesterificação do acetato de etila com metanol. Este estudo independe do catalisador empregado, do tipo de reator, e se presta a subsidiar a definição de parâmetros reacionais quando um catalisador adequado for identificado. Os resultados do presente trabalho são de fundamental importância para o estudo das propriedades de transesterificação de catalisadores uma vez que a reação do acetato de etila com metanol pode servir de modelo para diversos produtos incluindo biodiesel e ésteres para a indústria de solventes, polímeros, aromas e medicamentos.

\section{AGRADECIMENTOS}

Agradecemos ao Laboratório de Simulação de Processos e Catálise (LaSiP) e à CBMM pela utilização do software utilizado na simulação. E, a FUNARBE, FAPEMIG e CNPq, pelo suporte financeiro. 


\section{REFERÊNCIAS}

ALONSO, D. M. et al. Polarity of the acid chain of esters and transesterification activity of acid catalysts. Journal of Catalysis, v. 262, n. 1, p. 18-26, 2009.

CASTRO, C.S. Óxidos mistos de magnésio e alumínio contendo lítio e cálcio como catalisadores para transesterificação etílica. 2013 (Tese Doutorado). Universidade Federal de São Carlos - UFSCar, São Carlos-SP.

DEREVICH, I. V. et. al. Calculation of the Reforming of Oil Gas by Minimizing the Gibbs Free Energy. Theoretical Foundations of Chemical Engineering, v. 40, n. 2, p. 183-189, 2006.

ENCINAR, J. M. et al. Biodiesel fuels from vegetable oils: transesterification of cynaracardunculus $l$. oils with ethanol. Energy \& Fuels, 16, pp. 443-450, 2002.

LEAL, A. M. M. et al. Computational methods for reactive transport modeling: A Gibbs energy minimization approach for multiphase equilibrium calculations. Advances in Water Resources, v. 88, p. 231-240, 2016.

MARCINIUK, L. L. Síntese de titanatos alcalinos e avaliação na transesterificação catalítica. 2012 (Tese Doutorado). Universidade Federal de São Carlos - UFSCar, São Carlos-SP.

NÉRON, A.; LANTAGNE, G.; MARCOS, B. Computation of complex and constrained equilibria by minimization of the Gibbs free energy. Chemical Engineering Science, v. 82, p. 260-271, 2012.

PENG, Y. et al. Kinetic study of transesterification of methyl acetate with ethanol catalyzed by 4-(3-methyl-1-imidazolio)-1-butanesulfonic acid triflate. Applied Catalysis A: General, v. 466, p. 131-136, 2013.

PRAUSNITZ, J.M.; LICHTENTALER, R.N.; GOMEZ DE AZEVEDO, E. Molecular Thermodynamics of Fluid-Phase Equilibria, 2 ed. Englewood Cliffs : Prentice-Hall, 1986. $600 \mathrm{p}$.

TOMKE, P. D.; RATHOD, V. K. Ultrasound assisted lipase catalyzed synthesis of cinnamyl acetate via transesterification reaction in a solvent free medium. Ultrasonics Sonochemistry, v. 27, p. 241-246, 2015.

YANCY-CABALLERO, D.M. Método da minimização da energia de Gibbs para a modelagem do equilíbrio químico e de fases no processo reacional do biodiesel. Campinas: UNICAMP, Março. 2012. Dissertação de Mestrado. Departamento de Engenharia Química Faculdade de Engenharia Química. Universidade Estadual de Campinas.

YANCY-CABALLERO, D.M.,GUIRARDELLO, R. Thermodynamic simulation of transesterification reaction by Gibbs energy minimization. Fluid Phase Equilibria. V. 341, p.12-22, 2013. 


\title{
TRANSESTERIFICATION OF ETHYL ACEATATE WITH METANOL: THERMODYNAMIC ASSESSMENT
}

\author{
A. F. A. OLIVEIRA ${ }^{1,2}$, P. V. QUEIROZ ${ }^{1}$, C. H. F. CUNHA ${ }^{1}$, A. G. SATO ${ }^{1}$, F. A. \\ RODRIGUES $^{1}$ \\ ${ }^{1}$ Universidade Federal de Viçosa, Departamento de Química \\ ${ }^{2}$ E-mail: ana.f.alves@ufv.br
}

\begin{abstract}
Transesterification is one of the most important reactions of organic chemistry, and is responsible for the synthesis of several products in the most varied industrial sectors. The present study consists in evaluating the reaction behavior of the ethyl acetate and methanol transesterification reaction in order to obtain methyl acetate and ethanol using the Gibbs energy minimization approach for calculating chemical equilibrium. The simulation was performed in the software Aspen Plus 8.8 and for comparative purposes the reaction temperatures and molar ratios of reactant were varied. From the simulation results it is noted that the reaction temperature does not exert a great influence on the equilibrium composition. On the other hand, the thermodynamic equilibrium shows strong dependence on the molar ratio of reagents when the process is analyzed isothermally.
\end{abstract}

KEYWORDS: Transesterification; Simulation; Reaction Conditions; Thermodynamic. 